\title{
Blue hair pigmentation as inmunorelated adverse event in patient with non-small cell lung cancer (NSCLC) treated with anti- PDI immunotherapy: a
}

\section{case report}

\begin{abstract}
Currently there are new treatments available for the treatment of cancer. Consequently, there are new adverse reactions that should be known. Step by step, with the experience of using these new therapies, rare side effects are reported. In the case of immunotherapy, the appearance of immuno -related adverse effects may be associated with a longer survival and/or with a sustained tumor response over time. We report the case of a 69 year old male diagnosed with NSCLC, being treated with antiPD1 presented blue hair pigmentation as a side effect of treatment. It is a rare phenomenon, not previously described, that in our patient was associated with a longer survival despite the discontinuation of treatment.
\end{abstract}

Keywords: lung cancer, immunotherapy, immuno-related toxicity, hair pigmentation, blue hair
Volume 3 Issue 3 - 2018

\begin{abstract}
Amezcua-Hernández V, Doello González K García J,Valdivia Bautista J

Department of Medical Oncology, University Hospital Virgen de las Nieves, Spain
\end{abstract}

\begin{abstract}
Correspondence: Victor Amezcua Hernandez, Department of Medical Oncology, University Hospital Virgen de las Nieves, Spain, Tel +34958020234, Email euroncame@gmail.com
\end{abstract}

Received: April 26, 2018| Published: June 05, 2018

\section{Introduction}

Cancer is considered as a serious public health problem. According to the data of year 2016, around 1.7 million new cases of cancer are diagnosed in the United States. In a global manner, these cases, a $60 \%$ will have to receive systemic therapy while a $50 \%$ will be underwent to radiation treatment. The capillary toxicity, in the form of alopecia induced by cytotoxic therapy, is the most common side effect, more visible and the one that most affects quality of life of patients. ${ }^{1,2}$ There are cases in which the patient rejects the cytotoxic therapy - even in a curative context- because of the risk of potentially irreversible alopecia is associated to it. ${ }^{3}$ The new agents for the treatment of cancer, such as the new hormonal agents, immunotherapy or targeted therapy, in addition to radiation therapy can also cause hair toxicity, although in some cases it may be different.The case of the immunotherapy, and their application in recent years, has revealed new toxicities, many of them are not described in the scientific literature. We present the case of our patient, treated in the Hospital Virgen de las Nieves (Granada, Spain) Oncology Department with immunotherapy that firstly developed a capillary de pigmentation and then re-pigmentation to a colour in blue tones.

\section{Clinical case}

Man 69-year-old man. Diagnosed in August 2016 of non-small cell lung cancer stage IV by sub-pleural positive PET hyper metabolic nodules, in the right and middle upper lobe, osteolytic lesions in the costal arches and hepatic metastases. The EGFR, ALK and ROS1 mutational status study was negative. Immunohistochemical staining was performed on the piece, resulting in a $60 \%$ PDL1 positivity. Systemic treatment with chemotherapy was started with a platinumbased scheme (cisplatin + gemcitabine). He received only two cycles of doublet for bad tolerance. After the first cycle, he had to admitted to hospital due to pre-renal acute renal failure. After the second cycle, we had to enter the patient again due to vomiting grade IV (CTCAE v5.0) that made oral feeding impossible. For this reason, he continued exclusively with Gemcitabine reaching up to a total of 5 more cycles. In the PET revaluation progression of the liver disease was evidenced. We decided to start a second line of treatment with antiPD1 (Nivolumab).

The patient, in the beginning recovered the fallen hair as a result of the first doublet of cytostatics, without changes in the characteristics of which he had always had. After 4 cycles of Nivolumab, there was a depigmentation of it, becoming whiter than usual. No other skin lesions such as vitiligo appeared. After 9 cycles, the patient noticed that his hair was turning blue (Figure 1). The capillary transformation was monitored, increasing the blue tone up to the cycle 15. In parallel, the patient had a progressive neurological deterioration, cataloged after the corresponding study as immuno -related. For this reason, the treatment was discontinued. He remained in response for 4 more months of his lung disease, finally entering and dying 19 months after diagnosis.

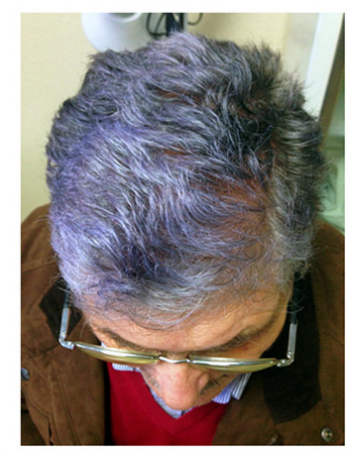

Figure I Hair immunorelated adverse effect. Blue pigmentation. 


\section{Discussion}

The capillary matrix of keratinocytes has a high mitotic activity, which makes it very vulnerable to the action of cytotoxic drugs. Changes in hair attributed to anticancer therapy occur in approximately $65 \%$ of patients receiving cytotoxic chemotherapy, $15 \%$ with targeted therapy (especially anti-EGFR), less than $2 \%$ of patients treated with immunotherapy and almost $100 \%$ of patients treated with radiation therapy in the head. ${ }^{4}$ In recent years, immunocheckpoints have become target of oncological therapy, producing an activation of the immune system promoting the detection and elimination of tumor cells. For the treatment of lung cancer, two antiPD1 drugs (nivolumab and pembrolizumab), antiPDL1 (atezolizumab) and the antiCTLA4 combination with antiPD1 (ipilimumab + nivolumab) are approved. ${ }^{5}$ There are phenomena of autoimmunity associated with them as side effect, having described serious adverse immunorelated effects. The most frequently affected organ is the skin and hair. ${ }^{6}$ At the level of the hair, the alopecia is the most frequent global effect, ${ }^{7}$ although there are also changes in the structure (becoming more brittle and brittle) or in color (both hyper and hypopigmentation). They are usually reversible after discontinuation of the treatment. Not only affect the hair of the patient, but also can affect the eyebrows, eyelashes or body hair. ${ }^{8}$ At the level of the skin, the most frequent side effect is the appearance of vitiligo, in a located or universal way. Hair pigmentation in blue tones is a very rare adverse effect. The mechanisms are unclear, although some hypotheses have been proposed. Some authors conclude that immunotherapy can inhibit proinflammatory cytokines such as (TNF alpha and betha, IL1 and IL2) that could act as negative regulators of melanogenesis, explaining the repigmentation. ${ }^{9,10}$ Other authors conclude that the melanocytes in the follicles could be activated by inflammation mediators such as cytokines or reactive oxygen species, explaining in many cases dermatological phenomena such as secondary folliculitis. ${ }^{11}$ At the level of the follicle, there could be interactions between drugs directed to immunocheckpoint and ion channels of copper and zinc because some antigen structure could be shared. In other diseases, a possible association between copper and zinc alterations and bluish hair pigmentation has been described. ${ }^{12}$ This issue is not well studied and could be a starting hypothesis to explain phenomena as rare as our patient. In a consistent manner with the literature, the fact that our patient developed immunorelated toxicity was associated with greater survival and a sustained response over time.

\section{Conclusion}

We report the case of a patient with pigmentation in blue hair tone, after starting a treatment with an immunotherapeutic drug antiPD1. It is a curious phenomenon, whose origin is not clear but which is probably a consequence of follicular toxicity as an immunorelated adverse event. The development of this capillary toxicity, in our case, was associated with a greater response and a longer survival. Until today, there are no other cases in the literature that document this phenomenon or possible scientific explanation to it. The communication of cases like this is necessary to increase clinical experience, generate research hypotheses and correlate with a greater number of patients if it is truly associated with a positive impact on overall survival.

\section{Acknowledgements}

None.

\section{Conflict of interest}

Author declares that there are no conflicts of interest.

\section{References}

1. Lemieux J, Maunsell E , Provencher L. Chemotherapy-induced alopecia and effects on quality of life among women with breast cancer: a literature review. Psycho-oncology. 2008;17(4):317-28.

2. Munstedt K, Manthey N, Sachsse S, et al. Changes in self-concept and body image during alopecia induced cancer chemotherapy. Supportive care in cancer. 1997;5(2):139-43.

3. Gandhi M, Oishi K, Zubal B, et al. Unanticipated toxicities from anticancertherapies: survivors' perspectives. Association of Supportive Care in Cancer. 2010;18(11):1461-8.

4. Freites MA, Shapiro J, Goldfarb S, et al. CME Part 1: Hair disorders in cancer patients. Journal of the American Academy of Dermatology. 2018;S0190-9622(18)30529-2.

5. Langer CJ. Emerging immunotherapies in the treatment of non-small cell lung cancer (NSCLC): the role of immune checkpoint inhibitors. Am J Clin Oncol. 2015;38(4):422-430.

6. Ribas A, Puzanov I, Dummer R, et al. Pembrolizumab versus investigator-choice chemotherapy for ipilimumab-refractory melanoma (KEYNOTE-002): a randomised, controlled, phase 2 trial. Lancet Oncol. 2015;16(8):908-918.

7. Zarbo A, Belum VR, Sibaud V, et al. Immune-related alopecia (areata and universalis) in cancer patients receiving immune checkpoint inhibitors. Br J Dermatol. 2017;176:1649-52.

8. Rivera N. Hair Repigmentation during immunotherapy treatment with an anti-programmed cell death 1 and anti- programmed cell death ligand 1 agent for lung cancer. JAMA Dermatol. 2017;153(11):11621165 .

9. LoveringS, MiaoW, BailieT, et al. Hair repigmentation associated with thalidomide use for the treatment of multiple myeloma. BMJ Case Rep. 2016; bcr2016215521.

10. DasanuCA, MitsisD, AlexandrescuDT. Hair repigmentation associated with the use of lenalidomide: graying may not be an irreversible process!. J Oncol Pharm Pract. 2013;19(2):165-169.

11. Cheng YP, Chen HJ, Chiu HC. Erlotinib-induced hair repigmentation. Int J Dermatol. 2014;53(1):e55-e57.

12. Tabatadze T, Zhorzholiani L, Kherkheulidze M, et al. Hair heavy metal and essential trace element concentration in children with autism spectrum disorder. Georgian Med News. 2015;(248):77-82. 